\title{
Niche-based process and neutral dynamics emerge the per capita ecological difference and equivalence among species at different spatio-temporal-environmental scales
}

\author{
takayuki yunoki ${ }^{1}$ \\ ${ }^{1}$ Universidad Autónoma del Beni José Ballivián
}

November 24, 2020

\begin{abstract}
Reconciling niche-based process and neutral dynamics in a portion of an infinite system, the regional species pool may be already not free parameter, and the divergent ecological-evolutionary mechanisms may operate consistently. The individual-based model was implemented in the two-dimensional grid with periodic boundary condition. The model was explored using a fixed speciation rate, and a range of system sizes, dispersal rates, environmental structures and initial conditions of regional species pool. The model communities in the center of system had a fixed population size, and approximated from an area encompassing independent biogeographic units to an area packed in a biogeographic unit with open boundary conditions, and presented the three environmental structures; four humps, linear and random. Across scenarios, the number of guilds in system achieved first to a stationary state; then, the species richness converged eventually to a dynamical equilibrium through speciation-extinction balance. In simulations, the per capita ecological difference among species only contributed to the probabilities of immigration success, so the weighted lottery process was more efficient and immediate at higher dispersal rates. The increase of functional redundancy in model communities suggested that the relative role of neutral dynamics increased in an area encompassing independent biogeographic units. The variation partitioning based on canonical analysis inferred that not only the neutral dynamics among the species of single guild, but also the competition-colonization trade-off among the species of more than two guilds with similar environmental optimum and different levels of specialization operated in the spatial structures found within and among patchy habitats. Ecologist to disentangle the influence of alternative processes must shift focus from the contribution of local competitions and regional dispersals to detecting the spatio-temporal-environmental scales on which the per capita ecological difference and equivalence among species are emerged through divergent ecological-evolutionary mechanisms.
\end{abstract}

\section{Introduction}

Ecologist traditionally relied on the deterministic process, such as environmental niche, and the coexistence of species was explained by a stationary state of ecologically different species (Gause, 1934; Hutchinson, 1957). Recently ecologist recognized also the role of stochastic process. The neutral dynamics were derived from the primary assumption of per capita equivalence among individuals and community saturation, such as ecological drift, random speciation and migration, and the coexistence of species was explained by a dynamical equilibrium of ecologically equivalent species in a set of local communities (Durrett \& Levin, 1996; Hubbell, 2001; Rosindell, Hubbell, \& Etienne 2011). In contemporary ecology, the reconciling of these divergent ecological-evolutionary mechanisms is a promising perspective to explain the origin and maintenance of biodiversity (Hubbell, 2001; Leibold \& Mcpeek, 2006; Munoz \& Huneman, 2016; Rosindell et al., 2011).

Neutral dynamics were based on individual-based model. The models were implemented mostly in the spatially-implicit and hierarchical conjecture, in that the extinction was balanced by the speciation under panmixis; while, the local extinction was balanced by the immigrats from a metacommunity. The regional 
dynamics were predicted by population size and speciation rate of metacommunity, and the local dynamics were predicted by further two parameters of population size and dispersal rate of local community (Hubbell, 2001; Etienne, 2005; Etienne, 2007; Etienne \& Oiff, 2004; Hankin, 2007; Munoz, Couteron, Ramesh, \& Etienne 2007). However, the spatially-implicit conjecture seems inconsistent in that the neutral dynamics assume panmixis in a metacommunity; whereas, dispersal-limitation to each local community embedded in it (Etienne, 2007). The spatially-explicit conjecture was one step approach allowing the speciation and dispersal-limitation across spatio-temporal scales. A metacommunity was usually a portion of an infinite system, and the models were implemented in a grid of local communities specifying neighbourhood or more general dispersal kernel (Durrett \& Levin, 1996; Hubbell, 2001; Chave \& Leigh, 2002; Chave, Muller-Landau, \& Levin 2002; Pigolotti \& Cencini, 2009).

Reconciling niche-based process and neutral dynamics in spatially-implicit conjecture, ecologist tried to explain the community assembly in successional patchy habitats, in that the vacant local community created by perturbation was initially colonized by the immigrants from a metacommunity under the ecological equivalence among species; then, the competitive exclutions were followed in each local community (Mouquet, Munguia, Kneitel, \& Miller 2003). In stable environment, the immigrants from a metacommunity was filtered along environmental gradients, and the coexistence of species in each local community was achieved under the per capita equivalence among individuals in homogeneous environment (Jabot, Etienne, \& Chave 2007; Janzen, Haegeman, \& Etienne 2015; Munoz, Ramesh, \& Couteron 2014; Munoz et al., 2018). In spatially-implicit conjecture; however, the regional dynamics were not predicted but usually specified by a fixed metacommunity. Strikingly, if the per capita ecological difference among species contributed to the competitions in each local community or the dispersals from a metacommunity; in general, the spatiotemporal scales on which the per capita ecological difference and equivalence among species contributed to metacommunity dynamics were credible assumpsions.

Reconciling niche-based process and neutral dynamics in spatially-explicit conjecture, a metacommunity may be a portion of an infinite system. The regional species pool may be already not free parameter, and the divergent ecological-evolutionary mechanisms may operate consistently at different spatio-temporalenvironmental scales. For instance, exploring the neutral model with nearest neighboring communities, an approximate scale on which individuals were expected to diffuse before speciation was predicted by a pair of speciation and dispersal rates (Cencini, Pigolotti, \& Muñoz 2012). In other words, the area of model communities must exceed an approximate scale predicted by these parameters to encompass at least one independent biogeographic unit. In the synthetic model with periodic boundary condition, where the environmental gradient repeats continuously across opposite sides of system, the relative role of neutral dynamics may increase in an area encompassing independent biogeographic units because the species richness but the number of functional groups (i.e. guilds) may increase through speciation.

In the present study, the individual-based model was implemented in the two-dimensional grid with periodic boundary condition. In simulations, the model parameters and the species properties in simulation outcomes were known by researcher, and the focus was to detecting the spatio-temporal-environmental scales on which the per capita ecological difference and equivalence among species were emerged through divergent ecological-evolutionary mechanisms. Exploring the model across a range of parameters, I proved specifically three hypotheses. First, the competitive exclusion may be advanced by the per capita ecological difference among species so the number of guilds may achieve first to a stationary state; while, the competitive exclusion may be retarded by the per capita ecological equivalence among species so the species richness may converge eventually to a dynamical equilibrium through extinction-speciation balance (Leibold \& Mcpeek, 2006). Second, the relative role of neutral dynamics may increase in an area encompassing independent biogeographic units. Third, the neutral dynamics may operate among the species of each guild in patchy habitats (Leibold \& Mcpeek, 2006; Economo \& Keitt, 2008). The trajectory and contrast between alternative processes establish the false premises that the niche-based process is only important in each local community; while, the neutral dynamics are only important in a set of local communities. I discuss finally the implication of false premises and consistent patterns observed in the present study for the performance of heuristic methods, the manner of their applications, and the interpretation of their results in field observations. 


\section{Materials and Methods}

\section{Simulation description}

The model was implemented in the rectangular two-dimensional grid with periodic boundary condition (Fig. 1). In simulations, the per capita ecological difference among species only contributed to the probabilities of immigration success; while, the per capita speciation, birth and death rates among individuals in local community were equivalent regardless of their identities.

The scenario was specified first by the standard parameters of neutral model; number of sites, speciation rate $\nu$ with point mutation mode of speciation and niche conservatism, number of individuals in regional species pool $J M$, dispersal rate $m$, and number of individuals in local community $J$. Then, a grid of local communities with coordinate $(x, y)$ and environmental gradient $E$ was specified in matrix landscape, and an initial condition of regional species pool; individuals code, species code, guilds code, their environmental optimum and tolerance was specified in matrix pool.t0 (Fig. 2a).

Based on a number of sites, a weights matrix for nearest neighboring communities with spatial weights $1 / 8$ was generated in a grid onto torus (matrix nb.mat ). The individuals of regional species pool were assigned to initial locations at random (matrix LC.t0 ). At each time step, all individuals were removed and replaced with probability $\nu$ by new species (matrix new.sp ). The matrix of remained individuals (matrix LC.t0.without.new.sp ) was standardized for each site by row sum (matrix LC.t0.RA ), and multiplied by matrix nb.mat to generate a set of probabilities of immigration success of individuals to each local community under neutral dynamics (matrix I.RA ). The matrix I.RA was multiplied element wise by a matrix of habitat associations of individuals to sites (matrixHA.siteBYind ), in that assigned weights depended on the value of probability density function for normal distribution given the mean as environmental optimum of individuals and the standard deviation as tolerance of individuals for the local environment of sites, and standardized for each site by row maximum. The element wise multiplication was standardized for each site by row sum to generate a set of probabilities of immigration success of individuals to each local community under environmental filtering (matrix EF ). The parents of each local community were chosen with replacement by a weighted lottery using a corresponding probability vector in $m$ x matrix EF $+(1-m)$ x matrix LC.t0.RA as the sum of immigrant (left term) and local birth (right term) parents (matrixLC.t1.without.new.sp ). Finally, the matrixLC.t1.without.new.sp was combined with the matrixnew.sp ; then, the extinct lineages of individuals were removed (matrix LC.t1 ). Also the matrix pool.t0 was adjusted for regional species pool (matrix pool.t1 ). The ancestors of new species were identified by the ancestor individuals codes and number of time steps in matrix LC.t1, and identified by these codes in matrix pool.t1 for individuals and species levels; while, the decendants from a common ancestor individual were identified by same code, and each lineage was grouped in a single column in matrixLC.t1 and row in matrix pool.t1 . The matrixpool.t1 and matrix LC.t1 were the input data for next time step (Fig. 2b).

I used a speciation rate $\nu=0.001$ and a number of individuals in local community $J=16$ individuals. The small system was simulated in a grid of $20 \times 20$ local communities using a $J M=6400$ individuals for two lower dispersal rates $m=(0.01,0.09)$. The large system was simulated in a grid of $40 \times 40$ local communities using $J M=25600$ individuals for the highest dispersal rate $m=0.81$. In small system, the environmental gradient $E$ presented three structures; random, one wave and 16 humps. In large system, the environmental gradient was quadrupled and presented three structure; random, two waves and 64 humps. In all cases, the environmental gradient was bounded between 0 and 1, and repeated continuously across opposite sides of system (Fig 1). The initial condition of regional species pool was varied by the number of functional groups $g$, habitat associations and population sizes of guilds. In small system, the environmental optimum of guilds was assigned following a uniform distribution between 0 and 1 , and the tolerance of guilds between 0 and 10. The population sizes of guilds were generated splitting $J M$ at $g-1$ points. These points were $g-1$ integers sampled from a uniform distribution between 1 and $J M-1$ and sorted by their values. The simulations in each combination of dispersal rate and environmental structure were started from the same set of five levels of $g=(1,8,40,160,500)$, five habitat associations and five population sizes of guilds except for the 
case of $g=1$ (i.e. the simulations were started from 25 habitat associations for which only one population size of a guild was possible). In large system, the initial condition of regional species pool was quadrupled. The simulations in each environmental structure were started from the same set of five levels of $g$ and five habitat associations of guilds; however, only one case of population sizes of guilds was explored because the computation in large system was intensive and time-consuming. So that 825 scenarios, $750=2 \times 3 \times 4 \times 5 \times 5+$ $2 \times 3 \times 1 \times 25$ were simulated in the small system with two lower dispersal rates and $75=1 \times 3 \times 5 \times 5$ in the large system with the highest dispersal rate.

In the neutral model with nearest neighboring communities and $\nu=0.001$, the approximate scales on which individuals were expected to diffuse before speciation were three for $m=0.01$, nine for $m=0.09$, and 28 for $m=0.81$ (Cencini et al., 2012). The model was explored using a fixed speciation rate, and a range of system sizes, dispersal rates, environmental structures and initial conditions of regional species pool. The model communities were a grid of $10 \times 10$ local communities in the center of system, and approximated from an area encompassing independent biogeographic units to an area packed in a biogeographic unit with open boundary conditions (i.e. the individuals diffused across borders of system were expected to originate new species at the opposite sides of model communities), and presented the three environmental structures; four humps, linear and random (Fig. 1). Arguing from the approximate scales, the model communities in large system with $m=0.81$ was packed nine times more compactly within a biogeographic unit than small system with $m=0.01$ in each environmental structure. The real ecosystem may be huge, and the extinction may be balanced by minimum speciation rate (Bell, 2003), and ecologist may study often an area packed much more compactly within a biogeographic unit. At present; however, the computation in larger system with lower speciation rate is not possible. The data analyses were performed on $\mathrm{R}$ version 3.6.3 (R Core Team, 2019) using the packages spdep (Bivand et al., 2019), vegan (Oksanen et al., 2019), PCNM (Legendre, Borcard, Blanchet, \& Dray 2012), randomizr (Coppock, Cooper, \& Fultz 2019), reshape2 (Wickham, 2017), dplyr (Wickham, François, Henry, Müller, \& RStudio 2019), entropart (Marcon \& Hérault, 2019), packfor (Dray, Legendre, \& Blanchet 2016), pgirmess (Giraudoux, Antonietti, Beale, Pleydell, \& Treglia 2018), and ape (Paradis et al., 2019). R sources are available in https://github.com/takayukiyunoki/spatialIBM.git.

Check convergence to a dynamical equilibrium

In initial simulations, I noticed that the number of guilds in system achieved first to a stationary state; then, the species richness achieved eventually to a dynamical equilibrium through speciation-extinction balance. Furthermore, setting the same seeds in random number generator, the simulation outcomes from alternative diversities; monodominance (i.e. all individuals of each guild were single species) and infinite (i.e. all individuals of each guild were different species) converged when the species of each guild in regional species pool were originated from the different common ancestor individuals in the simulation outcome from monodominance guilds (hereafter convergence time). The simulations reported here were started from monodominance guilds.

\section{Relative role of neutral dynamics increase in an area encompassing independent biogeographic units}

The species-neutral and functional diversities were calculated using the $q$ order two for the model communities at convergence time. The functional diversity was based on the similarity matrix between pairs of species calculated as the overlapping percentage of Gaussian functions (Marcon \& Hérault, 2015). Then, the functional uniqueness and redundancy proposed by Ricotta et al. (2016) were used to represent approximately the relative roles of alternative processes. In the model communities resulted with only one guild and more than two guilds, these measures were compared across ecological-evolutionary scales in each environmental structure.

\section{Neutral dynamics operate among the species of each guild in patchy habitats}

The variation partitioning based on canonical analysis (i.e. redundancy analysis; Borcard, Legendre, \& Gillet 2011; Peres-Neto \& Legendre, 2010) and the autocorrelation method (Diniz-Filho et al., 2012) were also used to infer the relative roles of alternative processes. In general, the applications followed Yunoki \& Torres (2016); Yunoki, Torres, Pouilly, \& Hablützel (2017); Yunoki, Torres, \& Cholima (2018); Yunoki, Torres, \& Cholima 
(2019).

The variation partitioning was applied for the model communities at convergence time. In model communities, the species response curves might be often unimodal, so that the species abundances data was Hellinger transformed prior to analysis and the first and second-order orthogonal environmental variables were used (Borcard et al., 2011; Gilbert \& Bennett, 2010). The standard forward selection procedure was used because the habitat associations among the species of each guild were equivalent and these species might have similarities regarding their environmental and spatial associations (Borcard et al., 2011; Peres-Neto \& Legendre, 2010). If more than two guilds were coexisted in model communities and if the pure environmental component was significant, the hierarchical guild structure was identified by the $k$-means partitioning of linear combination scores (scaling 1) and simple structure index criterion. If the hierarchical guild structure was significant, the principal coordinates of neighbour matrices (PCNM) were constructed to model the spatial structures within and among patchy habitats in the residual variation of species composition between sites. The portion explained by each component was obtained by the adjusted coefficient of determination in overall and hierarchical models, and its significance was estimated by a randomization test applying an alpha of 0.05 .

The functional uniqueness and redundancy of model communities were compared to the relative portions explained by environmental and pure spatial components in the total explaind variation of overall model; then, the total explained variation and these relative portions were compared between overall and hierarchical models. Furthermore, the number of guilds coexisted in model communities was compared to the number of habitat types identified by hierarchical guild structure.

In the model communities resulted with only one guild, the autocorrelation method was applied for the species abundances predicted by true spatial and false environmental components. In the model communities resulted with more than two guilds, this method was applied for the true environmental component of overall model, and the spatial structures found by hierarchical model in each environmental context. The correlograms were calculated using the number of distance classes computed by Sturges method in overall model; while, three or two distance classes in each environmental context. In all cases, the Mantel correlation between matrices of correlation coefficients and correlogram distances among species was tested against the null hypothesis of less than zero applying an alpha of 0.05. Appendix A presents the summary statics of scenarios.

\section{Results}

In small sytem, six scenarios started from one guild and the eleventh habitat association were collapsed, because the environmental tolerance of guild was nearly zero $(<0.0052)$. The results presented here were based on 819 simulation outcomes.

Across scenarios, the number of guilds in system achieved first to a stationary state; then, the species richness converged eventually to a dynamical equilibrium through speciation-extinction balance. The number of guilds achieved to a stationary state faster in higher dispersal rates (Fig. 3).

In the model communities resulted with only one guild, the functional uniqueness was zero; however, the relative portion explained by environmental component in total explained variation was positively biased in linear environmental gradient (Fig. 4a). The type I error of pure environmental component was inflated in these scenarios. The spatial and pure spatial components were always significant (Fig. 4b).

In the model communities resulted with more than two guilds, the functional uniqueness and redundancy presented the opposite pattern across ecological-evolutionary scales. The functional uniqueness increased in an area packed within a biogeographic unit, that was referred well by the relative portion explained by environmental component in the total explained variation of overall model (Fig. 5a). The power of pure environmental component increased in these scenarios. The spatial and pure spatial components were always significant (Fig. 5b).

If the pure environmental component of overall model was significant, the hierarchical guild structure and the spatial structures found within and among patchy habitats were usually significant (Fig. 5b). Further- 
more, the total explained variation and the relative portions explained by environmental and pure spatial components in total explaind variation were similar between overall and hierarchical models; however, the number of guilds coexisted in model communities was often larger than the number of habitat types identified by the $k$-means partitioning of linear combination scores (scaling 1) and simple structure index criterion (Fig. 5a).

In the model communities resulted with only one guild, the Mantel correlations of true spatial component approached to zero in an area packed within a biogeographic unit (Fig. 6a). They were always significant in an area encompassing independent biogeographic units, and vanished only in some scenarios for an area packed within a biogeographic unit (Fig. 6b). The Mantel correlations of false environmental component also approached to zero in an area packed within a biogeographic unit (Fig. 6a); however, they were often significant, and did not control correctly the false detection in linear environmental gradient (Fig. 6b).

In the model communities resulted with more than two guilds, the Mantel correlations of true environmental component negatively departed in an area packed within a biogeographic unit (Fig. 7a), and tended to emerge the pattern of spatial autocorrelations (Fig. 7b). The Mantel correlations of the spatial structures found by hierarchical model in each environmental context were often significant (Fig. 7b).

\section{Discussion}

Reconcilling niche-based process and neutral dynamics in a portion of an infinite system, the regional species pool was already not free parameter. In general, the divergent ecological-evolutionary mechanisms operated consistently, and emerged the per capita ecological difference and equivalence among species at different spatio-temporal-environmental scales as expectations.

First, the number of guilds in system achieved first to a stationary state; then, the species richness converged eventually to a dynamical equilibrium through speciation-extinction balance. The convergence to a dynamical equilibrium from any initial diversities was perspected in neutral spatially-explicit conjecture (Hubbell, 2001). I argued from the duality of neutral model (Cencini et al. 2012) that the simulations started from monodominance guilds might achieve to a dynamical equilibrium at convergence time because all walkers of each guild were coalesced or annihilated.

In simulations, the per capita ecological difference among species only contributed to the probabilities of immigration success, so the weighted lottery process in system was more efficient and immediate, and the functional uniqueness so the relative role of niche-based process in model communities increased at higher dispersal rates. However, if the ecological difference contributes to only competitions, or both competitions and dispersals, these patterns relative to dispersal rates may be altered.

Second, in the model communities resulted with more than two guilds, the increase of functional redundancy suggested that the relative role of neutral dynamics increased in an area encompassing independent biogeographic units. The pattern of system convergence suggested that the relative role of neutral dynamics might increase at broader temporal scale through speciation as well.

The third hypothesis was not completely true. In the model communities resulted with more than two guilds, the functional uniqueness and redundancy were refered well by the relative portions explained by environmental and pure spatial components in the total explained variation of overall model. Furthermore, the total explained variation and these relative portions were similar between overall and hierarchical models; however, the number of guilds coexisted in model communities was often larger than the number of habitat types identified by hierarchical guild structure. It was intuitively true that not only the neutral dynamics among the species of single guild, but also the competition-colonization trade-off among the species of more than two guilds with similar environmental optimum and different levels of specialization operated in the spatial structures found within and among patchy habitats.

The trajectory and contrast between alternative processes establish the false premises that the niche-based process is only important in each local community; while, the neutral dynamics are only important in a set of local communities (Leibold \& Mcpeek, 2006). The critic of variation partitioning based on canonical 
analysis was primary based on the false premises and the complexity to refer the overall parameters of selection strength and dispersal rate of simulations (Gilbert \& Bennett, 2010; Smith \& Lundholm, 2010); however, the neutral assumption can be broken by the difference of dispersal abilities, and the efficiency of weighted lottery process is dependent of dispersal rates as noted above. To compare the performance of existing approaches to disentangle the influence of niche-based process and neutral dynamics (e.g. generalised additive model and tree-based machine learning used in macroecology; Viana, Keil, \& Jeliazkov 2019), ecologist must shift focus from the contribution of local competitions and regional dispersals to detecting the spatio-temporal-environmental scales on which per capita ecological difference and equivalence among species are emerged. In this focus, the autocorrelation method (Diniz-Filho et al., 2012) and the analysis of functional ecology (e.g. Pillar \& Duarte, 2010) can be combined straightforward to infer divergent ecologicalevolutionary mechanisms.

In the present study, the first and second-order orthogonal environmental variables were used to model unimodal relationship. In the model communities resulted with only one guild, the functional uniqueness was zero; however, the relative portion explained by environmental component in total explained variation was positively biased in linear environmental gradient, and the type I error of pure environmental component was inflated in these scenarios. The species composition might be contrasted between opposite sides of model communities because of the approximate scales of three for $m=0.01$, nine for $m=0.09$ and 28 for $m=0.81$, and the phylogenetically closely related species might be clumped there. In the model communities resulted with more than two guilds, the functional uniqueness and redundancy were refered well by the relative portions explained by environmental and pure spatial components. The insufficiency of raw environmental variable to model unimodal relationship (Gilbert \& Bennett, 2010), and its dependence on environmental structures (Smith \& Lundholm, 2010) seemed to be solved mostly by the use of orthogonal environmental variables; furthermore, the neutral dynamics and competition-colonization trade-off were refered well in the spatial structures found within and among patchy habitats. Although the orthogonal environmental variables might increase the spurious correlation between species distribution and environmental structure across scenarios, this problem would be eventually corrected by the method proposed recently using a constrained randomization of environmental variables (Wagner \& Dray, 2015; Clappe, Dray, \& Peres-Neto 2018).

The performance of autocorrelation method (Diniz-Filho et al., 2012) was unexpected but interesting, and the spatial autocorrelation pattern of alternative processes reversed across ecological-evolutionary scales. The neutral dynamics always emerged the pattern of spatial autocorrelations in an area encompassing independent biogeographic units; while, the relative role of niche-based process increased and tended to emerge the pattern of spatial autocorrelations in an area packed within a biogeographic unit. It was intuitively true that the phylogenetically closely related species were clumped, and the neutral dynamics might vanish the pattern of spatial autocorrelations if the model communities were packed much more compactly within a biogeographic unit. Furthermore, the neutral dynamics through speciation as well as competition-colonization trade-off might emerge the pattern of spatial autocorrelations in patchy habitats.

In the fish communities of Bolivian Amazon lowlands, we observed the hierarchical guild structure across a range of spatio-temporal scales (Yunoki \& Torres, 2016; Yunoki et al., 2017; Yunoki et al., 2018; Yunoki et al., 2019). We presumed that only the neutral dynamics emerged the spatial structures among patchy habitats if all environmental factores were included in analysis, that the neutral dynamics did not emerge the pattern of spatial autocorrelations, and that the neutral dynamics should not be associated with functional species traits. The last argument is because of neutral assumption. The temporal structures found in the successional patchy habitats on a fine spatio-temporal scale usually presented autocorrelation patterns, and were accompanied by the seasonal and inter-annual change of functional species traits. We interpreted these patterns as environmental filtering. The spatial structures found among patchy habitats on a very broad spatial scale presented the autocorrelation pattern in turbid rivers but in varzea lakes, and the spatial structures found in turbid rivers and transparent black-clear water bodies but in varzea lakes were accompanied by the divergence of functional species traits (i.e. traits divergence pattern associated to hierarchical guild structure; see Pillar \& Duarte, 2010). Furthermore, the group of sedentary species was dominant in turbid rivers and transparent black-clear water bodies, and presented autocorrelation patterns there; while, the 
group of migratory species was dominant in varzea lakes, and did not present autocorrelation pattern there. We interpreted these patterns like the natural selection of sedentary species in the stable environment of turbid rivers and transparent black-clear water bodies as the species might have been adapted to a minor environmental factor missing from analysis, and the neutral dynamics of migratory species in the seasonal environment of varzea lakes (Yunoki et al., 2019); however, the natural selection of sedentary species in turbid rivers and transparent black-clear water was doubtful. Just as the patterns of nucleotide and amino acid substitution in population genetics (Holsinger, 2015), the natural selection may operate in the initial stage of diversification when the population sizes of each species may be huge; then, conserved for their descendants, and this expectation seems to be approximated by the mode of speciation and a range of initial conditions of regional species pool implemented here. The massive extinction of archaic faunas triggered by climate change during the Paleogene, and the roles of niche conservatism and environmental heterogeneity originated by principal geomorphological features (e.g. water types) for the origin of modern biodiversity during the Neogene were also perspected in the recent synthesis of biogeography of Neotropical freshwater fishes (Albert \& Reis, 2011). The simulations reported here were simple and unrealistic in many aspects; however, established the similar patterns with our field observations and seemed to allow their interpretations. The spatial structures and autocorrelation pattern found among patchy habitats of turbid rivers and transparent black-clear water bodies on a very broad spatial scale between north and south of Bolivian Amazon lowlands associated with the functional species traits of fish communities; furthermore, the autocorrelation patterns found there for the group of sedentary species but migratory species could be explained by the competition-colonication trade-off, and the speciations of sedentary species with niche-conservatism.

The niche-based process is deterministic and results first in a stationary state of guilds; while, the neutral dynamics are stochastic and result eventually in a dynamical equilibrium of species through speciationextinction balance. The relative role of neutral dynamics increases at broader spatio-temporal scales because the species richness but the number of guilds increases through speciation. The neutral dynamics among the species of single guild and the competition-colonization trade-off among the species of more than two guilds with similar environmental optimum and different levels of specialization operate in patchy habitats. The neutral assumption can be broken by the difference of dispersal abilities, and the efficiency of weighted lottery process is dependent of dispersal rates. The implication of present study is that the methodological advance and field studies to disentangle the influence of alternative processes must shift focus from the contribution of local competitions and regional dispersals to detecting the spatio-temporal-environmental scales on which the per capita ecological difference and equivalence among species are emerged through divergent ecological-evolutionary mechanisms.

Data accessibility

R sources are available in https://github.com/takayukiyunoki/spatialIBM.git. Appendix A presents the summary statics of scenarios. Bolivian Amazon lowland fish metacommunity data. Freshwater Metadata Journal 7: 1-6. http://dx.doi.org/10.15504/fmj.2015.7

References

Albert, J. S., \& Reis, R. E. (2011). Historial biogeography of Neotropical freshwater fishs. University of California Press

Bell, G. (2003). The interpretation of biological surveys. Pros R Soc, 270, 2531-2542. doi: $10.1098 / \mathrm{rspb} .2003 .2550$

Bivand, R., Altman, M., Anselin, L., Assunção, R., Berke, O., Bernat, A., .. Yu, D. (2019). spdep: Spatial Dependence: Weighting Schemes, Statistics and Models (R package version 1.1-3)

Borcard, D., Legendre, P., \& Gillet, F. (2011). Numerical ecology with R. Springer Science \& Business Media, NY

Cencini, M., Pigolotti, S., \& Muñoz, M. A. (2012). What Ecological Factors Shape Species-Area Curves in Neutral Models? PLoS ONE, 7(6), e38232. doi: 10.1371/journal.pone.0038232 
Chave, J., \& Leigh, E. G. (2002). A spatially explicit neutral model of beta-diversity in tropical forests. Theor Popul Biol, 62, 153-168. doi: 10.1006/tpbi.2002.1597

Chave, J., Muller-Landau, H. C., \& Levin, S. A. (2002). Comparing classical community models: theoretical consequences for patterns of diversity. Am Nat, 159, 1-23. doi: 10.1086/324112

Clappe, S., Dray S., \& Peres-Neto, P. R., (2018). Beyond neutrality: disentangling the effects of species sorting and spurious correlations in community analysis. Ecology, 99, 1737-1747. doi: https://doi.org/10.1002/ecy.2376

Coppock, A., Cooper, J., \& Fultz, N. (2019). randomizr: Easy-to-Use Tools for Common Forms of Random Assignment and Sampling (R package version 0.20.0)

Diniz-Filho, J. A. F., Siqueira, T., Padial, A. A., Rangel, T. F., Landeiro, V. L., \& Bini, L. M. (2012). Spatial autocorrelation analysis allows disentangling the balance between neutral and niche processes in metacommunities. Oikos, 121, 201-210. doi: 10.1111/j.1600-0706.2011.19563.x

Dray, S., Legendre, P., \& Blanchet, G. (2016). packfor: Forward Selection with permutation (Canoco p.46) (R package version $0.0-8$ )

Durrett, R., \& Levin, S. (1996). Spatial Models for Species-Area Curves. J. theor. Biol., 179, 119-127. doi: 10.1006/jtbi.1996.0053

Economo, E. P., \& Keitt, T. H. (2008). Species diversity in neutral metacommunities: a network approach. Ecol Lett, 11, 52-62. doi: 10.1111/j.1461-0248.2007.01126.x

Etienne, R. S. (2005). A new sampling formula for neutral biodiversity. Ecol Lett, 8, 253-260. doi: 10.1111/j.1461-0248.2004.00717.x

Etienne, R. S. (2007). A neutral sampling formula for multiple samples and an 'exact' test of neutrality. Ecol Lett, 10, 608-618. doi: 10.1111/j.1461-0248.2007.01052.x

Etienne, R. S., \& Olff, H. (2004). A novel genealogical approach to neutral biodiversity theory. Ecol Lett, 7, 170-175. doi: 10.1111/j.1461-0248.2004.00572.x

Gause, G. F. (1934). The Struggle For Existence. Williams and Williams

Gilbert, B., \& Bennett, J. R. (2010). Partitioning variation in ecological communities: do the numbers add up? - J. Appl. Ecol., 47, 1071-1082. doi: 10.1111/j.1365-2664.2010.01861.x

Giraudoux, P., Antonietti, J. P., Beale, C., Pleydell, D., \& Treglia, M. (2018). pgirmess: Spatial Analysis and Data Mining for Field Ecologists (R package version 1.6.9)

Hankin, R. K. S. (2007). Introducing untb, an R Package For Simulating Ecological Drift Under the Unified Neutral Theory of Biodiversity. Journal of Statistical Software, 22, 1-15. doi: 10.18637/jss.v022.i12

Holsinger, K. E. (2015). Lecture Notes in Population Genetics available at http://darwin.eeb.uconn.edu/eeb348-notes/Lecture-Notes-in-Population-Genetics.pdf under the Creative Commons Attribution License. Full terms at https://creativecommons.org/licenses/by/4.0/

Hubbell, S. P. (2001). The unified neutral theory of biodiversity and biogeography. Princeton University Press

Hutchinson, G. E. (1957). Concluding remarks. Cold Spring Harbor Symp Quant Biol, 22, 415-427. doi: 10.1101/SQB.1957.022.01.039

Jabot, F., Etienne, R. S., \& Chave, J. (2008). Reconciling neutral community models and environmental filtering: theory and an empirical test. Oikos, 117, 1308-1320. doi: 10.1111/j.2008.0030-1299.16724.x

Janzen, T., Haegeman, B., \& Etienne, R. S. (2015). A sampling formula for ecological communities with multiple dispersal syndromes. J. theor. Biol., 374, 94-106. doi: 10.1016/j.jtbi.2015.03.018 
Legendre, P., Borcard, D., Blanchet, F. G., \& Dray, S. (2012). PCNM: MEMspatial eigenfunction and principal coordinate analyses ( $\mathrm{R}$ package version $2.1-2$ )

Leibold, M. A., \& Mcpeek, M. A. (2006). Coexistence of the niche and neutral perspectives in community ecology. Ecology, 87, 1399-1410. doi: 10.1890/0012-9658

Marcon, E., \& Hérault, B. (2015). entropart: An R Package to Measure and Partition Diversity. Journal of Statistical Software, 67, 1-26. doi: 10.18637/jss.v067.i08

Marcon, E., \& Hérault, B. (2019). entropart: Entropy Partitioning to Measure Diversity (R package version $1.6-4)$

Mouquet, N., Munguia, P., Kneitel, J. M., \& Miller, T. E. (2003). Community assembly time and the relationship between local and regional species richness. OIKOS, 103, 618-626. doi: 10.1034/j.1600-0706.2003.12772.x

Munoz, F., Couteron, P., Ramesh, B. R., \& Etienne, R. S. (2007). Estimationg parameters of neutral communities: From one single large to several small samples. Ecology, 88(10), 2482-2488. doi: 10.1890/07-0049.1

Munoz, F., Grenié, M., Denelle, P., Taudière, A., Laroche, F., Tucker, C., \& Violle, C. (2018). ecolottery: Simulating and assessing community assembly with environmental filtering and neutral dynamics in R. Methods Ecol Evol, 9, 693-703. doi: 10.1111/2041-210X.12918

Munoz, F., \& Huneman, P. (2016). From the neutral theory to a comprehensive and multiscale theory of ecological equivalence. The Quarterly Review of Biology, 91, 321-342. doi: 10.1086/688098

Munoz, F., Ramesh, B. R., \& Couteron, P. (2014). How do habitat filtering and niche conservatism affect community composition at different taxonomic resolutions? Ecology, 95, 2179-2191. doi: 10.1890/13-0064.1

Oksanen, J., Blanchet, F. G., Friendly, M., Kindt, R., Legendre, P., McGlinn, D., .. Wagner, H. (2019). vegan: Community Ecology Package (R package version 2.5-6)

Paradis, E., Blomberg, S., Bolker, B., Brown, J., Claude, J., Cuong, H. S., .. de Vienne, D. (2019). ape: Analyses of Phylogenetics and Evolution (R package version 5.3)

Peres-Neto, P. R., \& Legendre, P. (2010). Estimating and controlling for spatial structure in the study of ecological communities. Glob Ecol Biogeogr, 19, 174-184. doi: 10.1111/j.1466-8238.2009.00506.x

Pillar, V. D., \& Duarte, L. S. (2010). A framework for metacommunity analysis of phylogenetic structure. Ecol Lett, 13, 587-596. doi: 10.1111/j.1461-0248.2010.01456.x

R Core Team (2019). R: A language and environment for statistical computing. R Foundation for Statistical Computing, Vienna, Austria. https://www.R-project.org/

Ricotta, C., de Bello, F., Moretti, M., Caccianiga, M., Cerabolini, B. E. L., \& Pavoine, S. (2016). Measuring the functional redundancy of biological communities: A quantitative guide. Methods Ecol Evol, 7, 1386-1395. doi: $10.1111 / 2041-210 X .12604$

Rosindell, J., Hubbell, S. P., \& Etienne, R. S. (2011). The Unified Neutral Theory of Biodiversity and Biogeography at Age Ten. Trends Ecol. Evol., 26(7), 340-348. doi: 10.1016/j.tree.2011.03.024

Smith, T. W., \& Lundholm, J. T. (2010). Variation partitioning as a tool to distinguish between niche and neutral processes. Ecography, 33, 648-655. doi: 10.1111/j.1600-0587.2009.06105.x

Viana, D. S., Keil, P., \& Jeliazkov, A. (2019) Partitioning environment and space in species-by-site matrices: a comparison of methods for community ecology and macroecology. bioRxiv preprint. Accessed November 3, 2020. doi:http://dx.doi.org/10.1101/871251

Wagner, H. H., \& Dray, S. (2015) Generating spatially constrained null models for irregularly spaced data using Moran spectral randomization methods. Methods Ecol Evol, 6, 1169-1178. doi: 10.1111/2041210X.12407 
Wickham, H. (2017). reshape2: Flexibly Reshape Data: A Reboot of the Reshape Package (R package version $1.4 .3)$

Wickham, H., François, R., Henry, L., Müller, K., \& RStudio (2019). dplyr: A Grammar of Data Manipulation ( $\mathrm{R}$ package version 0.8.4)

Yunoki, T., \& Torres, L. V. (2016). Fish metacommunity dynamics in the patchy heterogeneous habitats of varzea lakes, turbid river channels and transparent clear and black water bodies in the Amazonian lowlands of Bolivia. Environ Biol Fish, 99, 391-408. doi: 10.1007/s10641-016-0481-1

Yunoki, T., Torres, L. V., Pouilly, M., \& Hablützel, P. I. (2017). Comunidades ictícolas en diferentes tipos de aguas, Amazonía boliviana. Dissertation, I Congreso boliviano de ictiología. https://www.researchgate.net/publication/320716089_yunoki_bio-eco_oral

Yunoki, T., Torres, L. V., \& Cholima, R. B. (2018). A metacommunity ecological approach to understanding the community organization of fish in artificial ponds of the Mamoré River floodplain in the Amazonian lowlands of Bolivia. Environ Biol Fish, 101, 1329-1341. doi: 10.1007/s10641-018-0780-9

Yunoki, T., Torres, L. V., \& Cholima, R. B. (2019). Organización de las comunidades de peces en las tierras bajas de Amazonía boliviana. Dissertation, II Congreso boliviano de ictiología. https://www.researchgate.net/publication/337919664_YUNOKI_diversidad_oral

Figure legends

Figure 1. The environmental structures of model communities in a grid of $10 \times 10$ local communities in the center of small system. The environmental structure was quadrupled for large system.

Figure 2. A work flow script illustrating individual-based model. (a) parameters (b) first time step.

Figure 3. System convergence; one-dimensional scatter plots showing the time ratio to stationary guild number in the convergence time of species richness. The number of scenarios started from multiple guilds was provided following the text of axis-x in parentheses.

Figure 4. Summary statics of the model communities resulted with only one guild were compared across ecological-evolutionary scales in three environmental structures. (a) Functional uniqueness and redundancy were compared to the relative portions explained by environmental and pure spatial components in total explained variation. (b) Type I error rate of environmental and pure environmental components and power rate of other components. The number of model communities was provided following the text of axis- $\mathrm{x}$ in parentheses.

Figure 5. Summary statics of the model communities resulted with more than two guilds were compared across ecological-evolutionary scales in three environmental structures. (a) Functional uniqueness and redundancy were compared to the relative portions explained by environmental and pure spatial components in the total explained variation of overall model; then, the total explained variation and these relative portions were compared between overall and hierarchical models. The portions explained in hierarchical model was calculated for the scenarios in that the hierarchical guild structure was significant. vs.guild-habitat, the number of guilds coexisted in model communities was compared to the number of habitat types identified by hierarchical guild structure. (b) Power rate of all components in overall and hierarchical models. The number of model communities was provided following the text of axis-x in parentheses.

Figure 6. Performance of autocorrelation method in the model communities resulted with only one guild. (a) Mantel correlation of false environmental and true spatial components. (b) Mantel test against the null hypothesis of less than zero. The numbers of false environmental and true spatial components were provided following the text of axis-x in parentheses.

Figure 7. Performance of autocorrelation method in the model communities resulted with more than two guilds. (a) Mantel correlation of true environmental component in overall model and pure spatial components in each environmental context. (b) Mantel test against the null hypothesis of less than zero. The number of 
true environmental components in overall model and the number of environmental contexts in hierarchical model were provided following the text of axis- $\mathrm{x}$ in parentheses.

Competing Interests

I have no conflicts of interest to disclose.

Author Contributions

T.Y. developed the theoretical formalism, performed the numerical simulations and analytic calculations, and wrote the original draft and review.

Acknowledgements

I would like to thank editor in chief Prof. Jennifer Firn for the opportunity to revise my manuscript, also associate editor and two anonymous reviewers for the careful review and constructive suggestions.
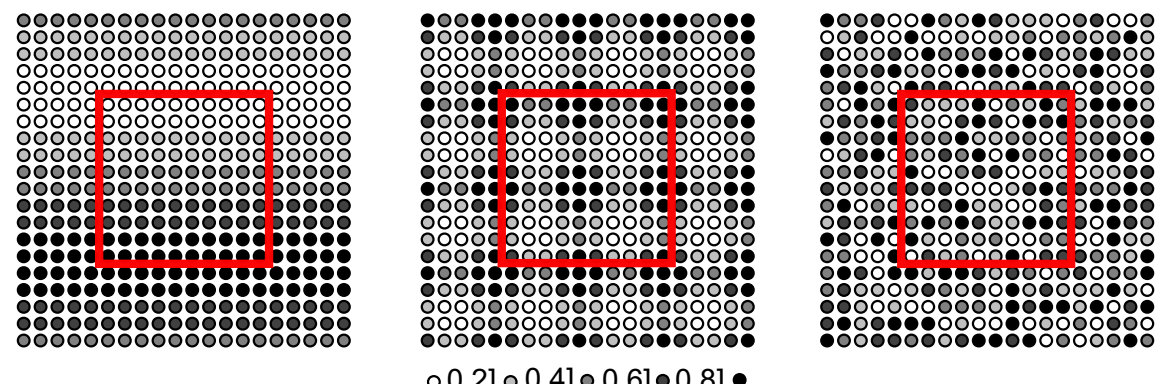

$\circ 0.2] \circ 0.4] \circ 0.6] \bullet 0.8] \bullet$ 
(a)

\section{parameters}

number of sites

speciation rate

number of individuals in regional species pool JM

dispersal rate

number of individuals in local community

$m$

landscape

coordinate $(x, y)$

pool.to environmental gradient $E$ optimum, tolerance
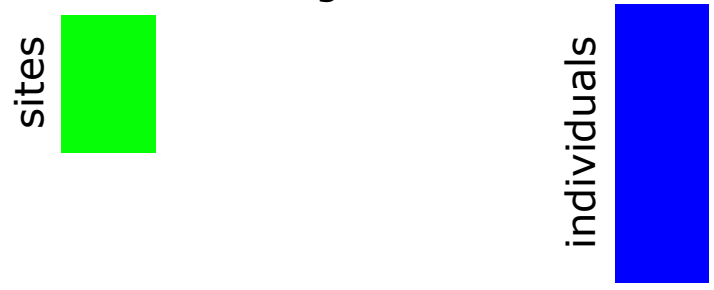

(b)

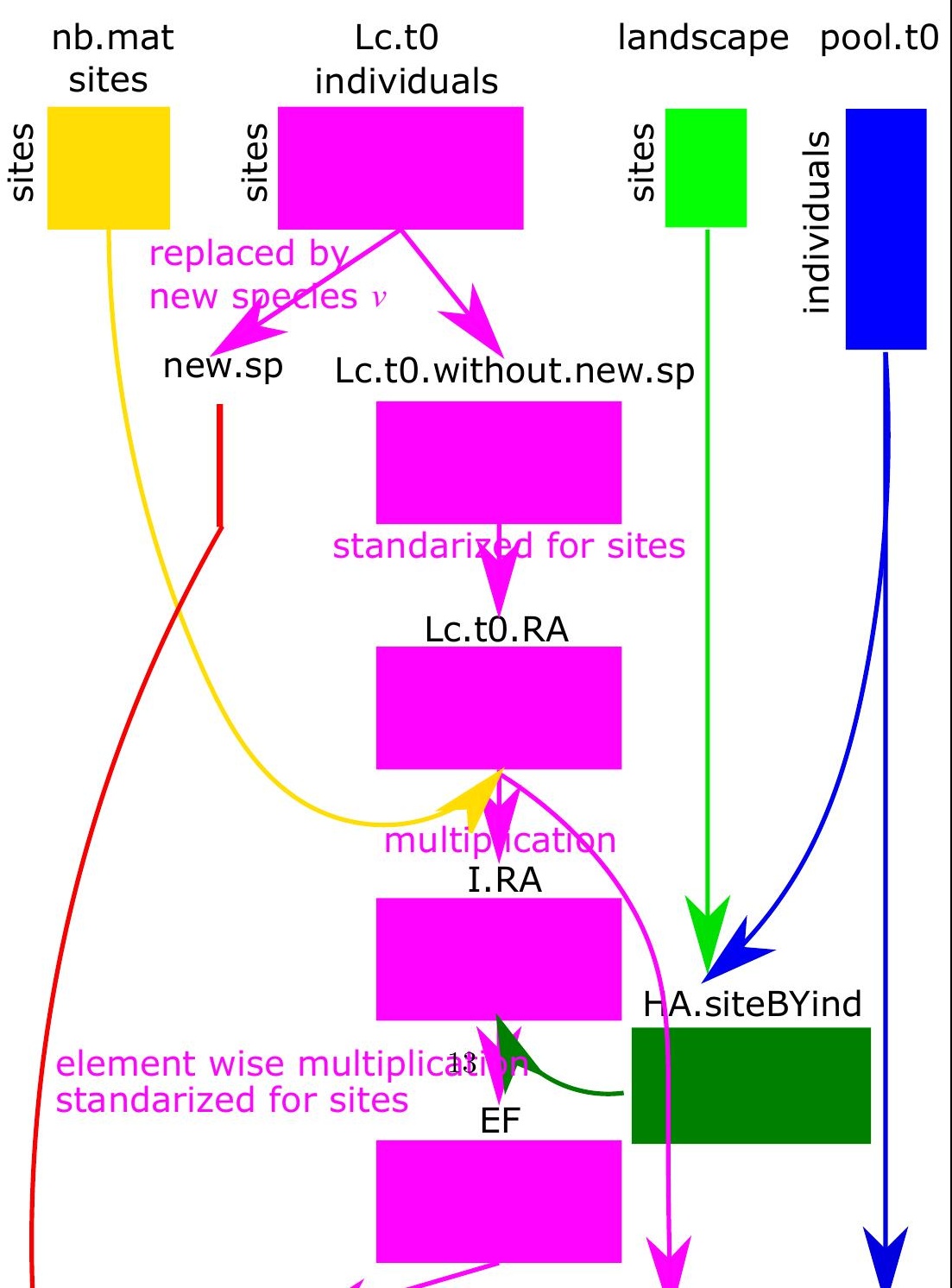



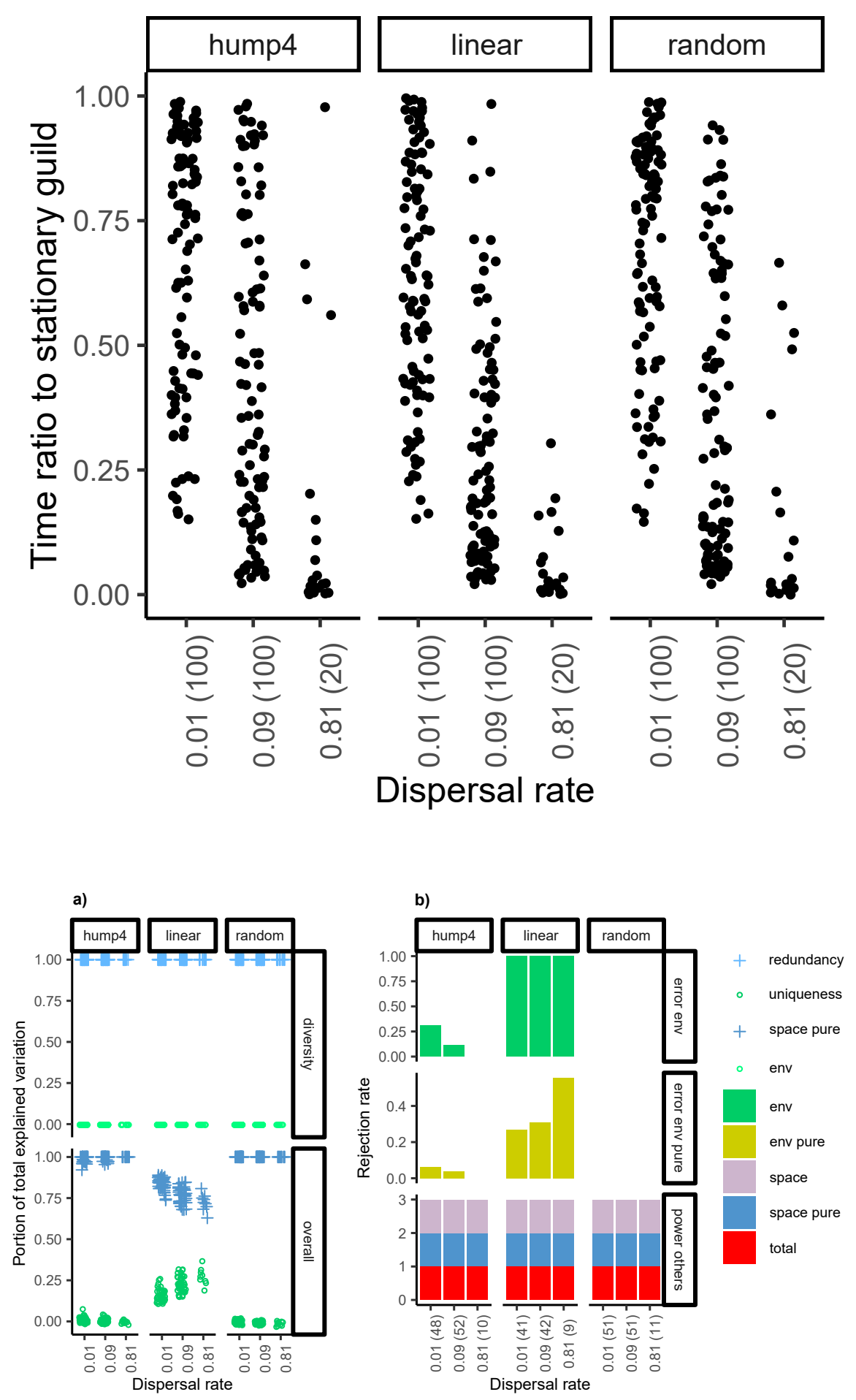

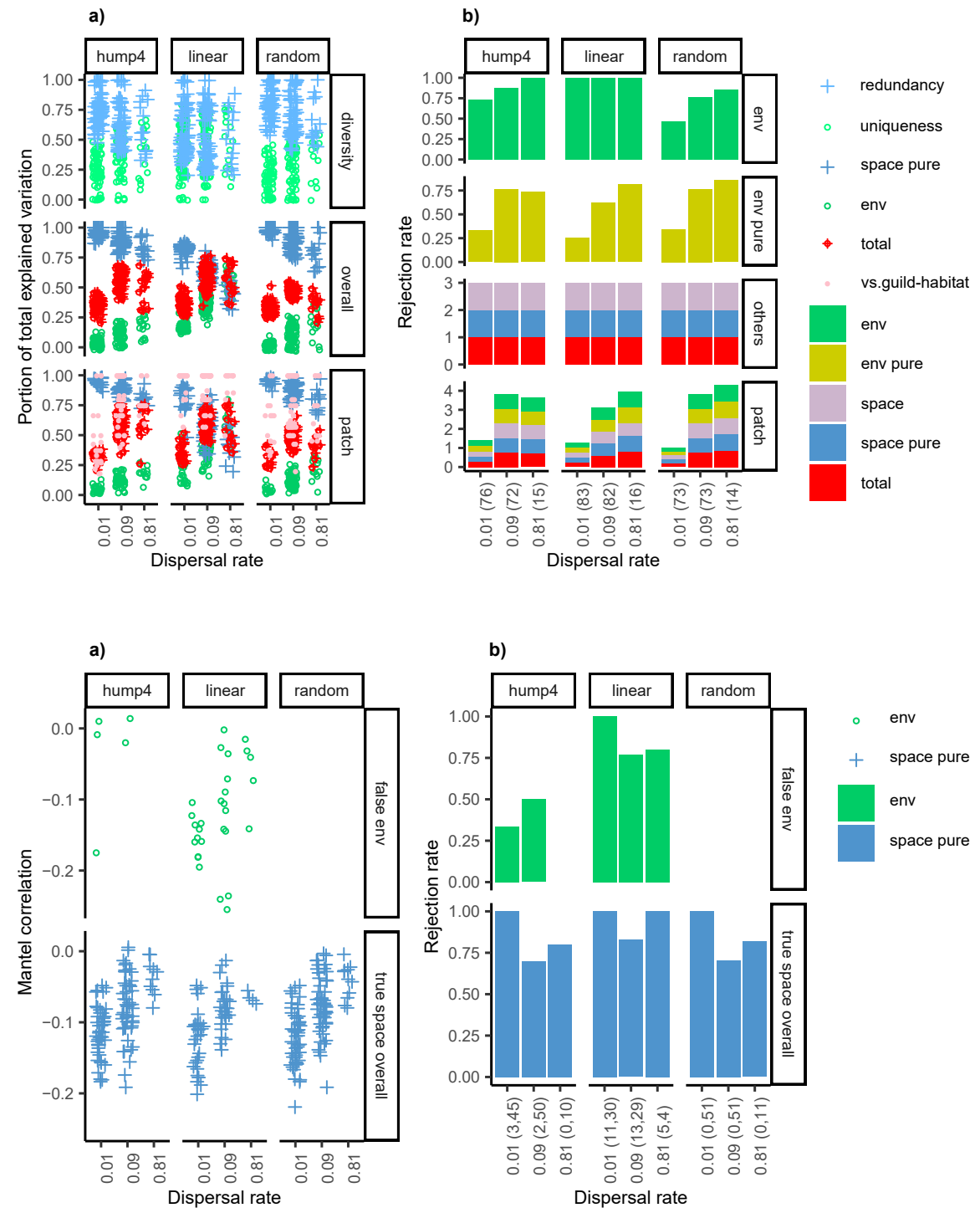

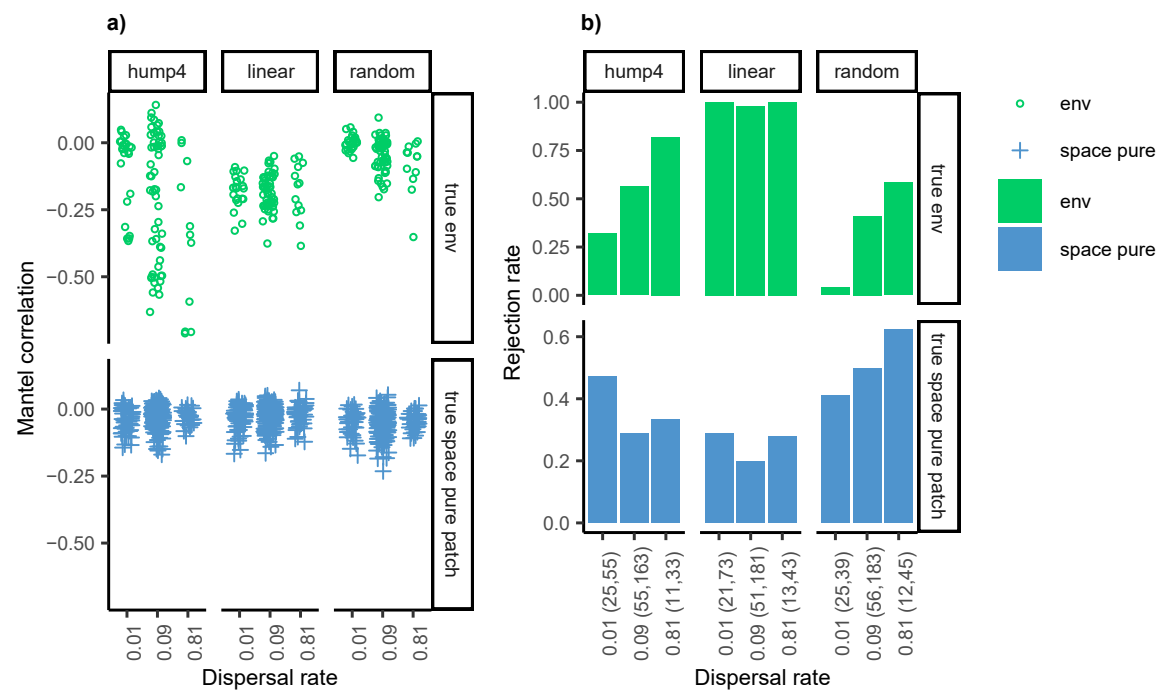